\title{
PHASE SPACE TOMOGRAPHY AT THE TESLA TEST FACILITY LINAC
}

\author{
M. Geitz, G. Schmidt, P. Schmüser*, DESY, D-22603 Hamburg
}

\begin{abstract}
The transverse phase space distribution of an electron beam can be determined with a quadrupole scan by measuring the resulting beam profiles via optical transition radiation and applying a tomographic reconstruction method. Phase space tomography has been successfully used at the TESLA Test Facility (TTF) linac equipped with a radio frequency photo-injector. Measurements of phase space distributions and emittances will be presented.
\end{abstract}

\section{INTRODUCTION}

To be usable as a driver for an envisaged Free Electron Laser in the VUV regime the TTF electron linac will have to provide $500 \mathrm{MeV}$ to $1 \mathrm{GeV}$ bunches with a charge of 1 $\mathrm{nC}$ and a normalized emittance in the order of $1-2 \pi \mathrm{mm}$ mrad. A laser-driven rf photocathode produces bunches with an rms length of 5 ps which are accelerated to about $5 \mathrm{MeV}$ in a $11 / 2$-cell rf cavity. The transverse phase space distribution and emittances of the electron beam are difficult to predict since they depend strongly on the density profile of the laser beam, on space charge forces and on the applied focusing by solenoid fields at the photoinjector. An experimental determination of the entire transverse phase space distribution can be accomplished by applying a quadrupole scan in combination with tomographic image reconstruction techniques [2]. For this purpose a set of quadrupoles is used to rotate the phase space distribution in well-defined angular steps between a reconstruction point $z_{0}$ in front of the quadrupoles and an observation point $z_{1}$ behind the quadrupoles. The transverse beam density distribution at the observation point is recorded by means of an optical transition radiation screen read out by a CCD camera. The horizontal and vertical beam profiles obtained at the different rotation angles allow to reconstruct the initial phase space distribution at $z_{0}$.

\section{PHASE SPACE TOMOGRAPHY}

The beam transport from $z_{0}$ to $z_{1}$ is described by a $4 \times 4$ transfer matrix $M$ whose elements are easily computed for given quadrupole settings. The measured horizontal charge density distribution can be expressed in terms of the horizontal phase space density $\rho_{1}$ at $z_{1}$ by the integral

$$
\begin{aligned}
p_{1}\left(x_{1}\right) & =\int \rho_{1}\left(x_{1}, x_{1}^{\prime}\right) d x_{1}^{\prime} \\
& =\int \rho_{1}\left(x_{1}, x_{1}^{\prime}\right) \delta\left(x_{1}-\tilde{x}_{1}\right) d \tilde{x}_{1} d x_{1}^{\prime} .
\end{aligned}
$$

\footnotetext{
* permanent adress: University of Hamburg, D-20146 Hamburg
}

Using the beam transfer matrix $M$, equation (1) can be rewritten in terms of the phase space distribution $\rho_{0}$ at the reconstruction point $z_{0}$

$$
p_{1, M}\left(x_{1}\right)=\int \rho_{0}\left(x_{0}, x_{0}^{\prime}\right) \delta\left(x_{1}-M_{11} x_{0}-M_{12} x_{0}^{\prime}\right) d x_{0} d x_{0}^{\prime} .
$$

We have added the index $M$ to $p_{1}\left(x_{1}\right)$ to indicate that the horizontal density distribution at $z_{1}$ depends explicitely on the transfer matrix $M$. In order to be compatible with the algorithms used in conventional computer tomography we introduce a "rotation angle" $\phi$ by the relations

$$
\cos \phi=\frac{M_{11}}{\sqrt{M_{11}^{2}+M_{12}^{2}}}, \quad \sin \phi=\frac{M_{12}}{\sqrt{M_{11}^{2}+M_{12}^{2}}} .
$$

This yields

$\tilde{p}_{1, \phi}(u)=\int \rho_{0}\left(x_{0}, x_{0}^{\prime}\right) \delta\left(u-x_{0} \cos \phi-x_{0}^{\prime} \sin \phi\right) d x_{0} d x_{0}^{\prime}$

with $u=x_{1} / \sqrt{M_{11}^{2}+M_{12}^{2}}$ and $\tilde{p}_{1, \phi}\left(u_{1}\right) \stackrel{(4)}{=}$ $\sqrt{M_{11}^{2}+M_{12}^{2}} \cdot p_{z, M}\left(x_{1}\right)$.

Hence we arrive at the conventional Cartesian rotation by scaling the measured intensity profiles with $\sqrt{M_{11}^{2}+M_{12}^{2}}$ and the $x$ coordinate with $1 / \sqrt{M_{11}^{2}+M_{12}^{2}}$. The distribution $\tilde{p}_{1, \phi}(u)$ has to be determined at narrow equidistant angular steps covering at least $180^{\circ}$ in order to permit a reconstruction of the distribution $\rho_{0}$ which is free from artifacts. In practice the full angular range is often not accessible and the step width may be too large. Interpolation and filtering techniques can be applied to improve the quality of such incomplete scans. A useful filter function is obtained by combining a frequency ramp function $|f|$ with a low-pass filter $L(f)$ [4]

$$
w(u)=\frac{1}{8 \pi^{2}} \int|f| L(f) \exp (i u f) d f .
$$

Here $f$ is a spatial frequency. The filtered profiles are given by

$$
p_{\phi}^{F}\left(x_{1}\right)=\int \tilde{p}_{\phi}\left(\tilde{x}_{1}\right) w\left(x_{1}-\tilde{x}_{1}\right) d \tilde{x}_{1} .
$$

The low-pass filter suppresses frequencies larger than the sampling frequency of the profiles and the ramp function enhances high spatial frequencies, i. e. profiles with strong curvature. The filtered profiles can now be backprojected to obtain the reconstruction $\rho_{0}^{R}\left(x_{0}, x_{0}^{\prime}\right)$ using

$$
\rho_{0}^{R}\left(x_{0}, x_{0}^{\prime}\right)=\int_{0}^{\pi} p_{\phi}^{F}\left(x_{0} \cos \phi+x_{0}^{\prime} \sin \phi\right) d \phi .
$$



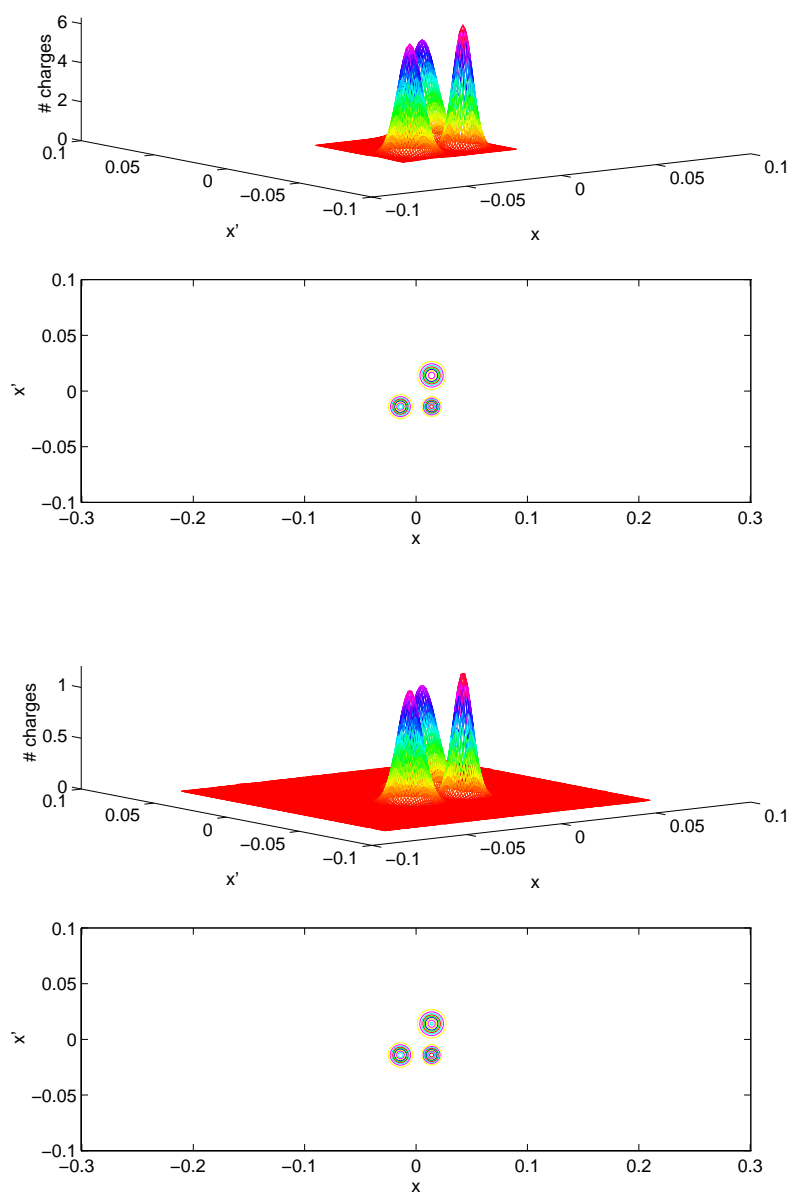

Figure 1: Result of a simulation using an asymmetric phase space distribution consisting of three peaks of different height and width. The agreement of the initial (upper graph) and reconstructed (lower graph) distribution is better than $1 \%$.

Figure 1 shows a simulation with an asymmetric phase space distribution consisting of three peaks of different height and width. The upper graph shows the initial distribution, the lower graph the reconstruction using 180 projections at a $1^{\circ}$ angular spacing. The reconstruction agrees with the initial distribution to better than $1 \%$.

\section{EMITTANCE AND OPTICS PARAMETERS}

The emittance is defined in terms of the variances of $x$ and $x^{\prime}$ by

$$
\varepsilon=\sqrt{\left\langle x^{2}\right\rangle\left\langle x^{\prime 2}\right\rangle-\left\langle x x^{\prime}\right\rangle}
$$

with

$$
\left\langle x^{2}\right\rangle=\frac{1}{N}\left(\sum_{i=1}^{N} x_{i}^{2}\right)-\frac{1}{N^{2}}\left(\sum_{i=1}^{N} x_{i}\right)^{2}
$$

$$
\begin{aligned}
\left\langle x^{\prime 2}\right\rangle & =\frac{1}{N}\left(\sum_{i=1}^{N} x_{i}^{\prime 2}\right)-\frac{1}{N^{2}}\left(\sum_{i=1}^{N} x_{i}^{\prime}\right)^{2} \\
\left\langle x x^{\prime}\right\rangle & =\frac{1}{N}\left(\sum_{i=1}^{N} x_{i} x_{i}^{\prime}\right)-\frac{1}{N^{2}}\left(\sum_{i=1}^{N} x_{i} \sum_{i=1}^{N} x_{i}^{\prime}\right)
\end{aligned}
$$

where $N$ denotes the total number of electrons of the beam. The optical functions $\alpha, \beta$, and $\gamma$ are given by

$$
x_{r m s}^{2}=\varepsilon_{r m s} \beta, x_{r m s}^{\prime 2}=\varepsilon_{r m s} \gamma \text { and }\left(x x^{\prime}\right)_{r m s}=\varepsilon_{r m s} \alpha .
$$

\section{SPACE CHARGE}

Linearized space charge forces are implemented into the beam transfer calculations. The lattice needed to compute the transfer matrix $M$ is subdivided into intervals of about $1 \mathrm{~mm}$. At these points, defocusing lenses are placed with
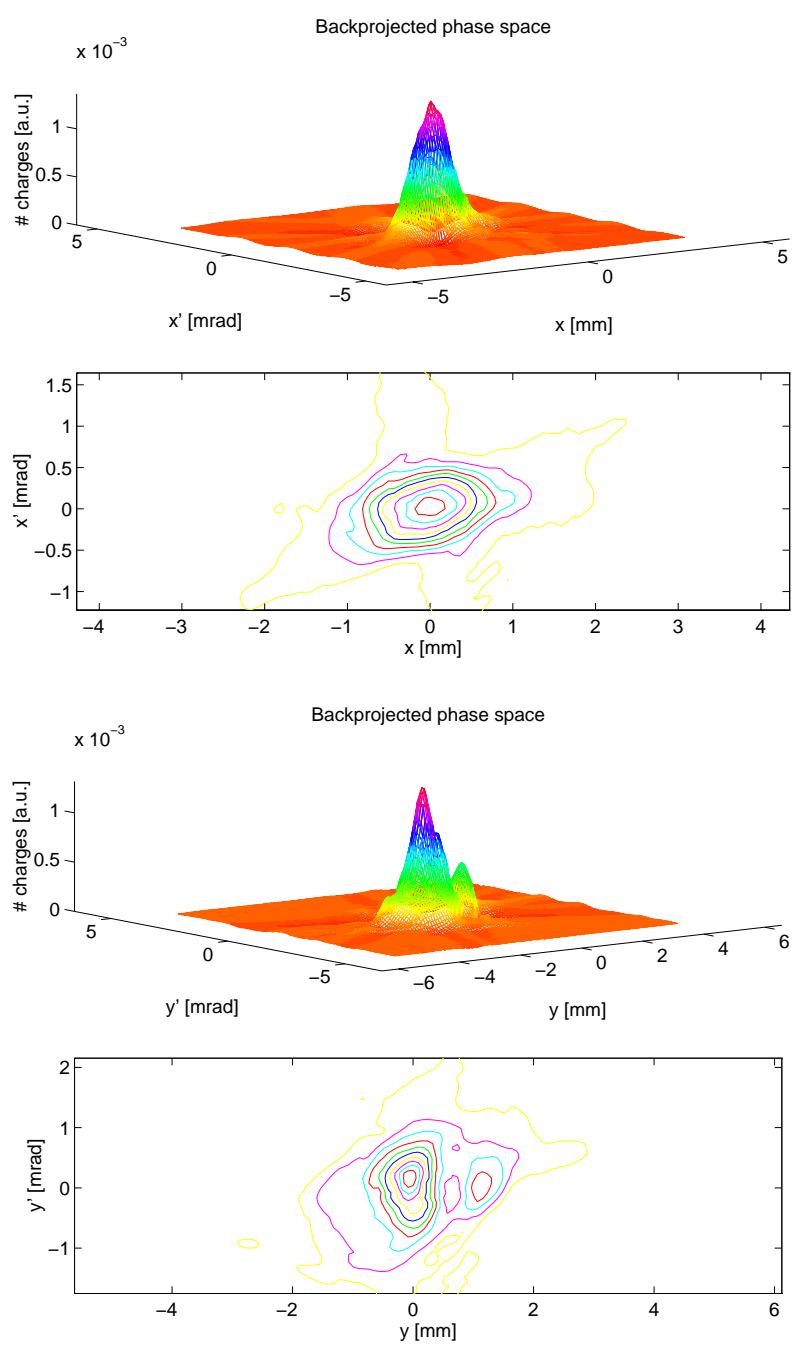

Figure 2: Reconstructed phase space density of the beam emitted by the photo injector, energy $16 \mathrm{MeV}$, charge $1 \mathrm{nC}$. 


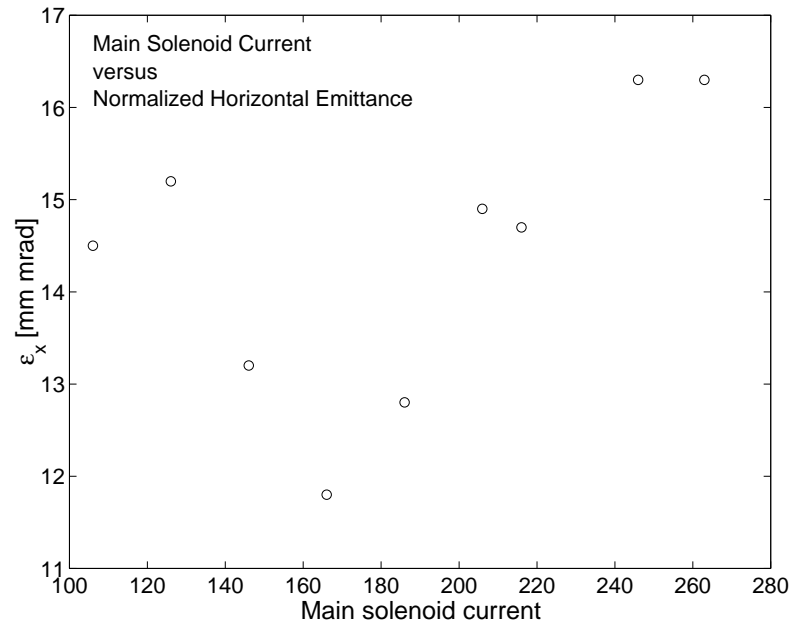

Figure 3: Normalized horizontal emittance versus the primary solenoid current. The secondary solenoid current is held at about $1 / 4$ of the primary current. Note the emittance assumes its minimum of $\varepsilon_{x}=11.8 \mathrm{~mm} \mathrm{mrad}$ at $I_{\text {prim }}=166 \mathrm{~A}, I_{\text {sec }}=41.5 \mathrm{~A}$. The systematic error of the emittance determination is about $30 \%$.

a strength

$$
\begin{aligned}
k_{x} & =\frac{2 N r_{e}}{\sqrt{2 \pi}} \frac{1}{\sigma_{x}\left(\sigma_{x}+\sigma_{y}\right) \sigma_{z} \gamma^{3}} \\
k_{y} & =\frac{2 N r_{e}}{\sqrt{2 \pi}} \frac{1}{\sigma_{y}\left(\sigma_{x}+\sigma_{y}\right) \sigma_{z} \gamma^{3}}
\end{aligned}
$$

where $\gamma$ denotes the Lorentz factor, $r_{e}$ the classical electron radius, $\sigma_{x}, \sigma_{y}$, and $\sigma_{z}$ the rms bunch dimensions. Gaussian-shaped bunches are assumed.

\section{MEASUREMENTS AT THE TTF LINAC}

A set of quadrupole scans has been performed behind the TTF photo injector and the capture cavity with a bunch charge of $1 \mathrm{nC}$ and a beam energy of $16 \mathrm{MeV}$. Here, space charge effects cannot be neglected. They are included in the analysis in linear approximation using formulae (13). The horizontal and vertical phase space density of the TTF beam is shown in Figure 2. The normalized emittances measured are

$\varepsilon_{x}=(5.5 \pm 2.5) \mathrm{mm} \mathrm{mrad}, \quad \varepsilon_{y}=(9.5 \pm 3) \mathrm{mm} \mathrm{mrad}$.

The beam emittance has been determined as a function of the strength of the gun solenoids. A variation with the magnetic field strength has to be expected because of the space charge forces acting on the charge distribution. These intrinsic forces are counter-balanced by the solenoid fields and an optimum setting has to be found for every bunch charge. The horizontal emittance is plotted in Figure 3 for different currents in the primary solenoid [5]. An normalized emittance of $\varepsilon_{x}=11.8 \mathrm{~mm}$ mrad has been obtained during the scan of the primary solenoid current between
100 and $270 \mathrm{~A}$. It should be noted that the emittance shown in Figure 2 is smaller because it was obtained after a careful optimization of all injector parameters [5].

\section{CONCLUSION}

The applicability of transverse phase space tomography has been demonstrated at the TTF electron linac. The technique permits a reconstruction of the transverse phase space distribution and allows the determination of the emittance and the optics parameters. Linearized space charge effects have been included in the beam transfer matrix.

\section{REFERENCES}

[1] TESLA - Collaboration, DESY-TESLA 95-01 (1995).

[2] O. R. Sander, G. N. Minerbo, R. A. Jameson and D. D. Chamberlin, Proc. of the Linear Accelerator Conference 1979, 1979.

[3] C. B. McKee, P. G. O'Shea, J. M. J. Maday, Nucl. Instr. Meth. A 358, (1995), 264 - 267.

[4] F. Natterer, The Mathematics of Computerized Tomography, Teubner, Stuttgart, 1996.

[5] S. Schreiber, private communication. 